\title{
Síndrome de Ondina (hipoventilación central congénita), una amenaza contra la vida al dormir
}

\author{
Lizeth Marín-Gómez'1 Liliana Adela Zuliani-Arango²
}

\section{RESUMEN}

El síndrome de Ondina, o síndrome de hipoventilación central congénita, es una enfermedad neurológica rara, donde hay fracaso en el control de la ventilación en el sistema nervioso central, llevando a la hipoxia e hipercapnia que pueden generar problemas del neurodesarrollo y, finalmente, ocasionar la muerte. Puede representar una situación muy dolorosa para los familiares de los pacientes que la padecen, despertando sentimientos difíciles de enfrentar, es por esto que es importante tener conocimiento acerca de esta condición para así impactar en la disminución de su incidencia.

\section{PALABRAS CLAVE}

Apnea Central del Sueño; Desarrollo Infantil; Sueño

\section{SUMMARY}

Ondina syndrome (congenital central hypoventilation) a threat against life when sleeping

Ondina syndrome, or congenital central hypoventilation syndrome, is a rare neurological disease in which there is a failure in the control of ventilation in the central nervous system,

Residente de Pediatría, Universidad de Antioquia, Medellín, Colombia.

Médica Neuropsicóloga Infantil, Magister en Salud Colectiva. Profesora Pediatría Social. Departamento de Pediatría y Puericultura. Facultad de Medicina. Universidad de Antioquia.

Correspondencia: Lizeth Marín Gómez; lizethmaring@hotmail.com

Recibido: julio 29 de 2018

Aceptado: noviembre 29 de 2018

Cómo citar: Marín-Gómez L, Zuliani-Arango LA. Síndrome de Ondina (hipoventilación central congénita), una amenaza contra la vida al dormir. latreia. 2019 Jul-Sep;32(3):243-247. DOI 10.17533/udea.iatreia.20. 
which leads to hypoxia and hypercapnia leading to neurodevelopmental problems and ultimately to death. It can represent a very painful situation for the family of patients who suffer from it, awakening feelings that are difficult to face. Thus, it is important to have adequate knowledge of this condition in order to have an impact on the decrease of its incidence.

\section{KEY WORDS}

\section{Child Development; Sleep; Sleep Apnea, CentraI}

\section{INTRODUCCIÓN}

El síndrome de Ondina es una enfermedad neurológica rara, caracterizada por el fracaso en el control de la ventilación de parte del sistema nervioso central. Esta condición puede llevar a la muerte debido a la hipoxia y la hipercapnia que se presenta en la persona que la padece (1).

Ondina, para la mitología germana era una ninfa. Las ninfas eran mujeres hermosas que vivían en fuentes de agua dulce y eran inmortales. Lo único que ponía en riesgo su felicidad eterna era enamorarse de un mortal y tener un hijo fruto de esa relación, lo que ocasionaría la pérdida de la inmortalidad. Según cuenta la leyenda, Ondina se enamoró de un mortal llamado Lawrence, se casaron y se prometieron fidelidad. Un año después de contraer matrimonio, Ondina dio a luz a un bebé, hijo de Lawrence, momento desde el cual comenzó a envejecer y a desvanecerse su belleza. Con el tiempo Lawrence perdió su interés en Ondina, hasta que un día mientras Ondina estaba caminando cerca de los establos, escuchó un ruido, se acercó para ver qué era y encontró a Lawrence recostado en los brazos de otra mujer.

Sorprenđida, lo señaló y pronunció su maldición: "Me juraste fidelidad por cada aliento que dieras mientras estuvieras despierto y acepté tu promesa, mientras te mantengas despierto, podrás respirar, pero si alguna vez llegas a dormirte, te quedarás sin aliento y morirás". En ese momento Lawrence se vio condenado a mantenerse despierto. Sin embargo, con el paso de los días, por el cansancio no aguantó más y se quedó dormido, no despertó jamás, cumpliéndose así la maldición de Ondina (2). Esta historia ha sido utilizada para dar al síndrome de hipoventilación central congénito el nombre de síndrome de Ondina, en el que se corre el riesgo de morir mientras se duerme.

A continuación, se presenta un caso clínico que puede ser de utilidad como un ejemplo para la identificación del síndrome.

\section{PRESENTACIÓN DEL CASO}

Paciente nacido a las 39 semanas de gestación, parto vértice espontáneo, 3.200 gr de peso y $52 \mathrm{~cm}$ de talla, aparentemente sano; tuvo una adaptación neonatal espontánea y fue dado de alta a las 24 horas de su nacimiento. Su madre en casa comenzó a notar un color diferente en la piel "moteado, con manchitas" y un quejido "como si le faltara oxígeno y morado alrededor de su boca”, por lo cual consultó a los 15 días de vida en el servicio de urgencias, donde encontraron un adecuado patrón respiratorio y una adecuada saturación del oxígeno. Sin embargo, fue hospitalizado con diagnóstico de ictericia.

Durante esta hospitalización, los médicos comenzaron a notar que en las horas de la noche y mientras el paciente dormía, presentaba esfuerzo respiratorio, cianosis, disminución en la saturación del oxígeno y períodos de apnea, por lo que tuvo que ser entubado y recibir manejo con ventilación mecánica.

Dentro de los exámenes diagnósticos se realizó una resonancia maognética cerebral, la cual mostró un pequeño sangrado cerebral, lo que llevó a pensar en maltrato. Sin embarogo, su madre lo negó rotundamente y con el tiempo fue descartado este diagnóstico.

En múltiples ocasiones tuvo extubación fallida, debido a que siempre manifestaba los mismos síntomas al dormir. Presentó además episodios convulsivos y en una ocasión paro cardiorrespiratorio que requirió reanimación avanzada.

Finalmente, se logró extubar y fue presentando una mejoría de los síntomas al ser manejado con ventilación de bipresión positiva (BPAP). Ya que este cuadro clínico solo se presentaba durante el sueño y se habían descartado otras posibles causas como el maltrato, alteraciones metabólicas, endocrinológicas, genéticas y neurológicas, los médicos sospecharon que se trataba del síndrome de Ondina. Se realizó 
una polisomnografía en la que se detectó hipoxia e hipercapnia que mejoraban al despertar o con el uso deI BPAP.

El paciente continuó su manejo con BPAP hasta que fue dado de alta a los 6 meses de vida con diagnóstico de síndrome de Ondina. Desde entonces y hasta este momento a sus 7 años de vida ha estado en manejo con BPAP mientras duerme, lo que le ha ayudado a mantener unos niveles de saturación de oxígeno permanentemente mayores a $92 \%$. Se encuentra al cuidado de una enfermera cada noche, quien monitoriza sus signos vitales, $y$ ha permanecido en seguimiento con especialistas para el manejo del síndrome y sus comorbilidades, como lo son las convulsiones y el retraso en el lenguaje que, aunque no se han evidenciado como consecuencia directa de su condición, pueden ser explicadas en cierta medida por la hipoxia repetida que pueden presentar estos pacientes.

Su madre ha vivido estos 7 años con el temor de perder a su hijo mientras duerme; los primeros días de vida no dormía bien pensando en que algo le iba a pasar, sin embaroo, con el tiempo comprendió el diagnóstico y los cuidados que debía tener. Por la hipoxia en su cerebro algunas conexiones cerebrales no se dieron o se presentaron con una maduración más lenta, haciendo que su desarrollo se viera afectado; por lo cual se demoró en caminar y en lograr algunos ítems del desarrollo en diferentes áreas: lentamente logró caminar y comunicarse con un lenguaje expresivo difícil de entender.

Ha estado en Prejardín y escuelas, pero con mucha angustia de la madre por temor a que se duerma y no despierte. Actualmente asiste a una institución educativa del municipio donde vive, pero su aprendizaje es lento y requiere más apoyo que otros niños, comprende lo que le enseñan pero a veces no logra retenerlo por mucho tiempo, lo que conocemos como memoria de largo plazo. Le gusta mucho la música y jugar en el computador de la casa, todo esto lo ha ayudado a ir mejorando un poco su lenguaje expresivo.

La presentación de este caso pretende ayudar a las madres $y$ al personal de la salud a identificar signos que puedan llevar al diagnóstico del síndrome de Ondina y evitar así que este sea el causante de una muerte súbita y, quizás, de un retardo en el desarrollo del lenguaje que puede mejorar con una buena estimulación.

\section{DISCUSIÓN}

El síndrome de Ondina es una entidad poco diagnosticada, que puede ser la causa no detectada de muchas alteraciones en el desarrollo de los niños y de las muertes infantiles de ocurrencia súbita.

El primer caso de síndrome de Ondina fue reportado en 1970 (3) y la incidencia fue estimada en 1 por cada 200.000 nacidos vivos en un estudio realizado en Francia (4). Sin embargo, debido a que aún se considera un síndrome raro $y$ poco diagnosticado, no existen hasta la fecha otros estudios que den cuenta de su incidencia. Su fisiopatología no es clara, estos pacientes generalmente tienen una ventilación adecuada mientras están despiertos, pero hay hipoventilación alveolar durante el sueño, con disminución del volumen corriente y de la frecuencia respiratoria. Los niños más gravemente afectados hipoventilan durante la viogilia y el sueño, además carecen de percepción de disnea, y cuando se enfrentan a problemas respiratorios, como infecciones, no pueden aumentar la ventilación para satisfacer las demandas, lo que puede empeorar el cuadro clínico (5).

Es una enfermedad de transmisión autosómica dominante que ha sido relacionada con mutaciones en el gen PHOX2B, cromosoma 4p12 (6). Este gen también juega un papel importante en la regulación de la migración de células de la cresta neural y el desarrollo del sistema nervioso autónomo, es por esto que los pacientes con síndrome de Ondina pueden presentar otras enfermedades que resultan de aberraciones en la migración, crecimiento o diferenciación de las células de la cresta neural, como por ejemplo, la enfermedad de Hirschsprung, que está presente en aproximadamente $16 \%$ de los pacientes (5).

Desde los comienzos de la vida, incluso durante la gestación, el ser humano va adquiriendo un conocimiento, el cual se ve influenciado por estímulos tanto internos como externos. Esta habilidad de ir madurando y adaptando sus diferentes funciones físicas, psicológicas, funcionales, sociales y culturales se conoce como neurodesarrollo, período que pasa de la dependencia del recién nacido a la autonomía del adolescente, para luego encontrarse con sus propias realidades $y$ proyectos vitales. El neurodesarroIlo durante los estadios tempranos de la vida se ve influenciado por múltiples factores ambientales que 
determinan fenómenos en el sistema nervioso central de diferenciación, mielinización, arborización dendrítica y sinaptogénesis.

Si una persona durante cualquier etapa de dicho ciclo vital sufre estrés o una situación que pueda afectar esta maduración, se presenta un retraso en la adquisición de habilidades motoras, cognitivas como el lenguaje, la memoria y la atención y comportamentales.

Estudios realizados por Vannuci y Perlman en 1997, hablan de cómo la hipoxia neonatal puede afectar entre un 2 y $4 \%$ de cada 1.000 nacidos vivos y llevar a una encefalopatía hipóxica que afectará el desarrollo de sus habilidades motoras, cognitivas o psicológicas de forma permanente en un $25 \%$ de los casos (7).

Un estudio realizado en México por la Facultad de Psicología y Medicina de la Universidad Autónoma de San Luis Potosí entre 1995 y 1998, concluyó que la hipoxia ya sea prenatal, perinatal o postnatal, genera un gran riesgo en el desarrollo por un déficit en el tejido neuronal y que la manifestación más frecuente es un retraso en el área de la motricidad gruesa y fina, según las áreas de desarrollo de Gesell, además del compromiso del coeficiente intelectual por alteraciones en el desarrollo del lenguaje expresivo (8).

El estudio concluye que la encefalopatía hipóxico-isquémica puede llevar a alteraciones en la adquisición del lenguaje expresivo más que el compromiso de la habilidad de comprensión del lenguaje; lo cual, según ellos, es de buen pronóstico. No obstante, tener dificultades en la expresión verbal influye en el coeficiente intelectual, ya que muchas tareas para evaluarlo son de respuestas verbales (8). Este retraso se vio en el niño descrito previamente, lo que da cuenta de que el diagnóstico de esta condición debe ser rápido para evitar que la hipoxia cerebral cause daños permanentes en el cerebro del niño, y tratar de limitar las consecuencias en las funciones cognitivas y comportamentales.

Como esta enfermedad no tiene un signo característico, su diagnóstico es de exclusión, requiriendo fundamentalmente la presencia de hipoventilación durante el sueño $\left(\mathrm{PaCO}_{2}>60 \mathrm{mmHg}\right)$, inicio durante los primeros meses de vida $y$ ausencia de una enfermedad del tronco del encéfalo, neuromuscular, pulmonar, metabólica, cardíaca u otra que pueda explicar el cuadro. Solo se requieren pruebas diagnósticas para excluir otras causas según las sospechas, algunos diagnósticos diferenciales son: malformaciones o tumores del tronco encefálico, infecciones del sistema nervioso central, enfermedades neuromusculares, síndrome de Moebius, metabolopatías, cardiopatías y anomalías de la vía aérea, entre otras (9). El sueño se convierte en un elemento fundamental para su reconocimiento, es por esto que vigilar el sueño y escuchar los síntomas que relatan las madres son primordiales para su diagnóstico.

Su manejo es multidisciplinar, dirigido a mantener una adecuada ventilación según los requerimientos del paciente, evitando la hipoxia secundaria a la hipoventilación durante el sueño. Su pronóstico ha venido mejorando al conocer un poco más de esta entidad y, en cuanto mayor conocimiento se tenga de esta, menores secuelas como el retraso en el neurodesarrollo y el crecimiento se van a presentar en niños con esta condición.

\section{CONCLUSIÓN}

El síndrome de Ondina es una enfermedad rara que representa un reto diagnóstico, por lo que conocer un poco más de ella ayudará a su oportuno reconocimiento, mejorando así la calidad de vida de estos pacientes y evitando muertes de manera súbita sin un diagnóstico oportuno.

Iniciar tempranamente un acompañamiento multidisciplinar puede hacer que la madre se sienta más segura en su crianza y no solo pensar en la supervivencia de su hijo, sino en todos los aspectos importantes de su desarrollo. Un buen vínculo afectivo y unas buenas pautas de crianza pueden darle al niño esa resiliencia necesaria para salir adelante con esta situación tan difícil.

\section{AGRADECIMIENTOS}

Al joven con síndrome de Ondina y a su familia por contarnos su historia $y$ permitirnos compartirla. Al grupo de puericultura de la Universidad de Antioquia por estimularnos a escribir.

\section{CONFLICTOS DE INTERESES}

Ninguno por declarar. 


\section{REFERENCIAS BIBLIOGRÁFICAS}

1. Zaidi S, Gandhi J, Vatsia S, Smith NL, Khan SA. Congenital central hypoventilation syndrome: An overview of etiopathogenesis, associated pathologies, clinical presentation, and management. Auton Neurosci. [Internet]. 2017;210:1-9. [consulted 2018 May 15] available from: http://dx.doi.org/10.1016/j.autneu.2017.11.003.

2. Varios. Leyenda: La maldición de Ondina. Octubre 2014. Narradores del misterio. [Internet] 2014; octubre 23. [Consultado 2018 mayo 15]. Disponible en: http://narradoresdelmisterio.net/leyenda-la-maldicion-de-ondina/.

3. Robert B. Mellins, Henry H. Balfour, Gerard M. Turino, Robert W. Winters. Failure of automatic control of ventilation (ondine curse). 1970;49(6).

4. Trang H, Dehan M, Beaufils F, Zaccaria I, Amiel J, Gaultier C. et al. The French Congenital Central Hypoventilation Syndrome Registry. Chest [Internet]. 2005;127(1):72-9. [consulted 2018 May 15] Available from: $\quad$ http://linkinghub.elsevier.com/retrieve/pii/ S0012369215323746.

5. Healy F, Marcus CL. Congenital central hypoventilation syndrome in children. Paediatr Respir Rev. 2011;12(4):253-63. DOI 10.1016/j.prrv.2010.10.001.

6. Amiel J, Laudier B, Attié-Bitach T, Trang H, De Pontual L, Gener B, et al. Polyalanine expansion and frameshift mutations of the paired-like homeobox gene PHOX2B in congenital central hypoventilation syndrome. Nat Genet. 2003;33(4):459-61. DOI 10.1038/ ng1 130 .

7. Vannuci RC, PerIman JM. Interventions for Perinatal Hypoxic-Ischemic Encephalopathy. Pediatrics. 1997;100(6):1004-14.

8. Ibarraran M, González Y, González C, Hernández JF. Influencia de la hipoxia perinatal sobre el desarroIlo en la etapa preescolar. Rev. Mex. Neuroci.. 2001; 2(5):281-87.

9. Orvay JAC, Pons M. Síndrome de Ondine : diagnóstico y seguimiento. An Pediatr (Barc). 2005;63(5):426-32. 